\title{
The detection of the early stages of ageing in an LDPE + graphite composite by comparison of dielectric responses induced by sinusoidal and triangular signals
}

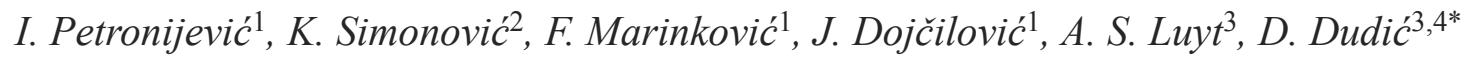 \\ ${ }^{1}$ University of Belgrade - Faculty of Physics, Studentski trg 12-16, 11001 Belgrade, Serbia \\ ${ }^{2}$ Faculty of Mechanical Engineering, Bogišičeva 8, 1000 Ljubljana, Slovenija \\ ${ }^{3}$ Department of Chemistry, University of the Free State (Qwaqwa Campus), Private Bag X13, 9866 Phuthaditjhaba, South \\ Africa \\ ${ }^{4}$ University of Belgrade - Vinča Institute of Nuclear Sciences, P.O. Box 522, 11001 Belgrade, Serbia
}

Received 11 March 2014; accepted in revised form 25 May 2014

\begin{abstract}
This study describes the possibility of dielectric characterization of the initial stages of ageing in an low-density polyethylene (LDPE) + graphite composite, which is not possible using the standard method of dielectric spectroscopy. It is shown that the differences between the delay angles, $\Delta \varphi=\varphi_{\mathrm{TRI}}-\varphi_{\mathrm{SIN}}$, obtained using triangular and sinusoidal excitations on the composite samples, shows a maximum, and at the same time the position of this maximum shows more sensitivity to changes in the electrical properties of the material caused by ageing than other dielectric parameters. In order to clarify the applied methodology, a comparative analysis of the dielectric properties of other polymers poly(vinyl chloride) (PVC) and poly(vinyl alcohol) (PVA) and a conductive polymer composite (LDPE + carbon black) with respect to the application of sinusoidal and triangular electrical signals was carried out. Based on the presented results, we believe that the position of the peak in the frequency spectra of the difference between the delay angles obtained by using triangular and sinusoidal signals may be a suitable parameter for the dielectric characterization of polymeric materials.
\end{abstract}

Keywords: polymer composites, triangular wave signal, dielectric, delay angle, ageing

\section{Introduction}

Various forms of degradation of polymeric materials and their composites represent an important factor that reduces their usability. The most common causes of changes in the properties of polymeric materials are: application of mechanical stress [1]; elevated temperature $[2,3]$; exposure to electromagnetic radiation of high energy $[4,5]$; presence of slowly varying electric fields [6]; contact with aggressive chemicals [7]. The changes in polymeric materials resulting from aforementioned factors can be classified into two groups. The first group consists of chemical changes such as oxidation processes, changes in molecular weight or other chemical reactions, and the second group involves the physical changes in the structure of the polymer or polymer composite, including phase transitions, changes in crystallinity, rearrangement of the amorphous phase and redistribution of fillers [8]. In most cases the physical and chemical processes occur together and affect each other.

A number of standards were developed that prescribe the methodology of investigation of degradation effects on the physico-chemical properties of these materials. Regardless of their application, polymeric materials are expected to have good

\footnotetext{
${ }^{*}$ Corresponding author, e-mail: ddudic@vinca.rs

(C) BME-PT
} 
mechanical properties, even after ageing. Among other properties such as chemical resistance, toxicity, optical properties and thermal stability, changes in electrical properties are important for polymer materials that are used in electronics or as electrical insulating materials. It has been shown that in cases where changes of electrical properties due to ageing are not of primary importance, their changes can still be correlated with, for example, changes in mechanical properties [9]. Most of the methods that examine the properties of the material show a relative measurement error of about $1 \%$, but in some cases this error can be much higher as is the case with stress-strain measurements or lower in density measurements. This measurement error is not a problem when examining the effect of ageing, which significantly changes the properties of the material. There is however a need to detect the initial stages of changes in material properties, which are important in, for example, electrical insulation materials, where the measuring sensitivity of the applied method becomes extremely important.

Electrical measurements have an important place in the field of materials characterization, and the measurement of the frequency spectra of AC conductivity provides more information than the $\mathrm{DC}$ conductivity measurement. DC measurement gives only one value, resistivity or conductivity, while dielectric measurements provide two parameters, such as amplitude of conductivity and delay angle, and also their frequency dependence. The dielectric measurement can also be improved by changing the shape of the excitation signal, which is what is presented in this article. The application of the sine wave electrical test signal in the investigation of the dielectric properties of polymers is a standard procedure in polymer science [10-12]. The other waveforms of a test signal are rarely used in the analysis of the electrical properties of polymers [13, 14], but they are used for the testing of energy transformers as well as for various electronic and mechanical tests [15-18]. It is well known that the dielectric relaxations in polymers occur over broad intervals of frequencies and/or temperatures, which sometimes makes difficult to precisely establish the dielectric parameters and the exact position of the processes (they are simply too broad to be noticed). The application of sinusoidal excitation allows the presentation of $\mathrm{AC}$ conductivity (admittance) and $\mathrm{AC}$ resistance (impedance) in a complex mathemat- ical form. The real and imaginary parts of admittance and impedance, and the complex permittivity $\left(\varepsilon^{*}=\varepsilon^{\prime}-i \varepsilon^{\prime \prime}\right)$ and angle $\delta$ (usually $\tan \delta$ ) are the dielectric parameters that are commonly used to describe the dielectric relaxation in polymers. In some cases the dielectric relaxation of polymers is clearly observed in the temperature and frequency dependencies of electrical modulus $\left(M^{*}=1 / \varepsilon^{*}\right)$, as well as appropriate derivations of the $\mathrm{AC}$ conductivity [19] or permittivity [11].

In the present study we used, besides the sine wave signal, a triangular test signal to investigate the early stages of ageing in an LDPE + graphite composite which was subjected to high oxygen pressure at room temperature. It was shown that the difference between the delay angles obtained by applying different excitations waveforms, triangular and sinusoidal, shows a maximum whose position on the frequency scale was very sensitive to changes in the electrical properties of the composites. In order to describe the applicability of the method to other polymer systems, we also analyzed some typical polymers (PVC and PVA) and a conductive polymer composite (LDPE + carbon black (CB)).

\section{Experimental}

\subsection{Materials}

PVC - The poly(vinyl chloride) sample was a commercial PVC cling film obtained from Shanghai StarLight Plastics Limited.

PVA - Merck-Alkaloid, art.821038.

LDPE - PE552, HIP Pančevo, Serbia, $M_{\mathrm{w}}=$ 110000 and $\rho=0.922 \mathrm{~g} / \mathrm{cm}^{3}$.

The carbon black (ellipsoidal shape, diameter 3$5 \mu \mathrm{m}$ ) and graphite flakes (about $10 \mu \mathrm{m}$ ) were obtained from the Laboratory for Thermal Engineering and Energy, Vinča Institute of Nuclear Sciences, Serbia.

\subsection{Samples preparation}

PVC samples of $210 \times 150 \mathrm{~mm}$ and thickness $d=$ $0.01 \mathrm{~mm}$ were cut from the middle of the sheets.

The poly(vinyl alcohol) sample was obtained from an aqueous PVA solution (1.5 mass\%). The polymer was dissolved in boiling distilled water and mixed at $6000 \mathrm{rpm}$ for 120 minutes. In order to get solid films, the solution was placed into a Petri dish and dried in a vacuum oven at $40^{\circ} \mathrm{C}$ for $24 \mathrm{~h}$. The sample was in the form of disk with diameter $D=$ $53 \mathrm{~mm}$ and thickness $d=0.11 \mathrm{~mm}$. 
LDPE + $20 \mathrm{wt} \%$ carbon black and LDPE + $30 \mathrm{wt} \%$ graphite composites were obtained in a Haake rheometer at $433 \mathrm{~K}$ for $10 \mathrm{~min}$. The isotropic sheets were prepared by compression molding at $460 \mathrm{~K}$ and a pressure of $1.75 \mathrm{MPa}$ for $5 \mathrm{~min}$, followed by quenching in water at room temperature. The LDPE + graphite composites were gamma irradiated in a ${ }^{60} \mathrm{Co}$ radiation facility, in air at room temperature, at a dose rate of $6 \mathrm{kGy} / \mathrm{h}(100$ and $200 \mathrm{kGy})$. The accelerated ageing was performed at $22^{\circ} \mathrm{C}$ in an Emmerson single vessel oxygen apparatus at $0.5 \mathrm{MPa}$. Samples of $50 \times 50 \mathrm{~mm}$ and thickness $d=$ $1 \mathrm{~mm}$ were cut from the middle of the sheets.

Electrical measurements were made more than 30 days after the mentioned treatments of the composites. Electrical contacts on the samples were made by fine copper powder.

\subsection{Methods}

The dielectric measurements were performed on a Tektronix TDS 2022B oscilloscope. An Agilent 33220A $20 \mathrm{MHz}$ function/arbitrary wave form generator was used as the voltage source. An amplitude of $7.8 \mathrm{~V}$ was applied and the frequency range was $0.005 \mathrm{~Hz}-160 \mathrm{kHz}$. The sample cell was housed in a Faraday cage and measurements were done at room temperature (normal pressure and 50\% relative humidity). The experimental set-up is shown in Figure 1.

In the measurement procedure, the electrical current through the sample was monitored via the resistor $R$ in Figure 1 (capacity less than $10^{-11} \mathrm{~F}$ ). The presence of this resistor might have induced distortion of the triangular signal applied to the sample. For this reason, the values of $R$ were changed in such a way to ensure that the voltage drop across the resistor did not exceed $1 \%$ of the input voltage of the function generator. The electrical current through

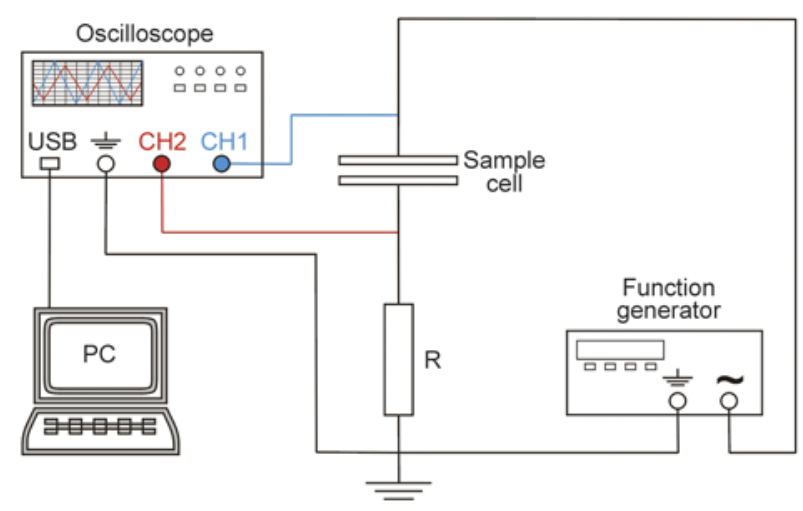

Figure 1. Block diagram of the experimental set-up the sample cell was calculated as $I(\mathrm{t})=U_{\mathrm{CH}_{2}}(t) / R$, while the voltage on the sample was determined as $U_{\mathrm{S}}(t)=U_{\mathrm{CH}_{1}}(t)-U_{\mathrm{CH}_{2}}(t)$. The reported results were obtained after the correction $I_{\mathrm{S}}(t)=I_{\mathrm{M}}(t)-I_{\mathrm{B}}(t)$ was applied, where $I_{\mathrm{S}}(t)$ is the current through the sample, $I_{\mathrm{M}}(t)$ the current measured with a sample in the cell, and $I_{\mathrm{B}}(t)$ the current in the empty cell without the upper cell electrode. The value of the current at each particular frequency $(f)$ was determined as the average value over the time interval that lasted at least $10 / f$. Relative values of the standard deviation of the obtained parameters were up to $2 \%$, and they did not change significantly with respect to the change in input signals. Electrical DC conductivity measurements were performed using an Agilent 4339B high resistance meter at room temperature, and applying an electric field of $100 \mathrm{~V} / \mathrm{mm}$ for $20 \mathrm{~min}$.

The results of measurements with sinusoidal excitation were related to the well-known current phase diagram in Figure 2a $\left(I_{0}\right.$ is the amplitude of the current). In contrast to the current amplitude obtained by application of the sinusoidal voltage, the amplitudes of the current obtained by application of a triangular waveform are always in phase with the voltage amplitude. Because of that, the delay angle was determined under the condition $I\left(t_{2}\right)=V\left(t_{1}\right)=0$ as shown in Figure 2b. The amplitude of the conductivity was defined as $Y_{0}=I_{0} / U_{0}$ for both waveforms, where $U_{0}$ and $I_{0}$ are the maximum values of the measured voltage and current, respectively. Data processing was performed in a script program originally designed for this purpose.

\section{Results and discussion}

\subsection{Dielectric response of PVC, PVA and LDPE + CB}

A comparison of the dielectric properties of polymers with the appropriate RC circuit models has already been presented in the literature [10-12]. We presented in the appendix of this paper an analysis of the electrical responses of serial and parallel RC circuits due to sinusoidal and triangular voltage excitations. Comparison of the dielectric response of RC circuits to sine and triangular excitation indicates that the angle of the triangular signal delay is always greater than or equal to the appropriate angle obtained by applying sinusoidal signals $(\Delta \varphi(f)=$ $\left.\varphi_{\text {TRI }}-\varphi_{\text {SIN }} \geq 0\right)$. One reason for this is that the response (current) of RC circuits to sine (voltage) 


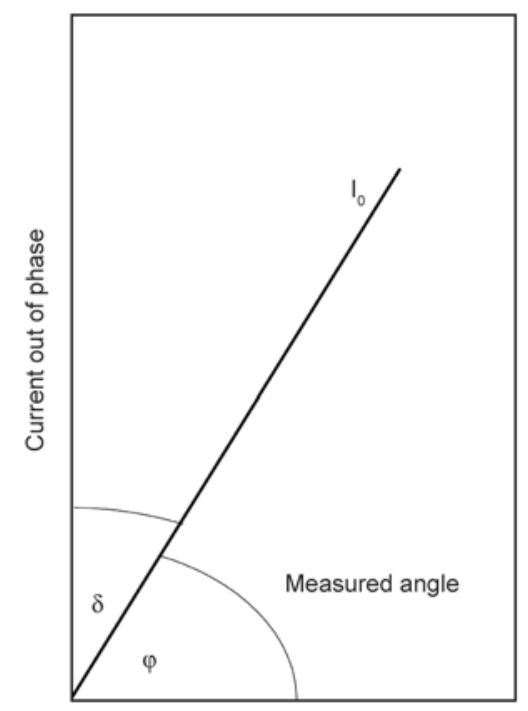

a)

Crrent in phase

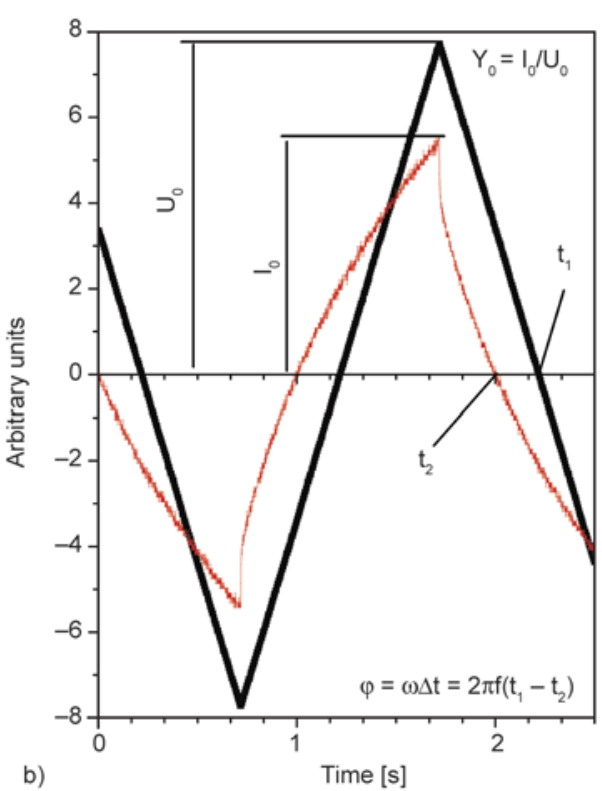

b)

Time $[\mathrm{s}]$

Figure 2. a) Current phase diagram for sinusoidal excitation; b) the response of PVA to triangular excitation at $0.5 \mathrm{~Hz}$

excitation also has a sinusoidal shape with a unique, time-independent delay angle, while the response of the RC circuits to triangular excitation has a complex frequency-dependent form, the amplitude of which is always in phase with the excitation. The phase deviation of the measured current of triangular excitation, due to the existence of a capacity, is the largest in the intersection of the measured current with the time axis (Figure 2b). This is used to determine delay angle for this form of excitation. In addition, numerical analysis shows that in the cases of ideal serial and parallel RC circuits there is a well defined peak of the function $\Delta \varphi(f)$ (Figures 9a and 10a). The position of the maximum in the difference between the delay angles in the frequency spectrum, $\Delta \varphi(f)=\varphi_{\mathrm{TRI}}-\varphi_{\mathrm{SIN}}$, obtained using triangular and sinusoidal excitation for ideal electrical circuits, can be related to the RC constant of the circuits (fo in Table 1). This gave us the idea to use the frequency, that corresponds to the maximum of $\Delta \varphi(f)$, to describe the electrical properties of polymers. In this section, the behaviour of the real polymer systems was tested on two polymers, PVC as a representative electrically non-conductive polymer, PVA that can then be considered as a semiconductor, and an LDPE $+20 \mathrm{wt} \% \mathrm{CB}$ composite with relatively high electrical conductivity.

Figure 3 shows the results obtained after the application of sinusoidal and triangular signals to the PVC sample. The loss tangent of PVC (Figure 3a)
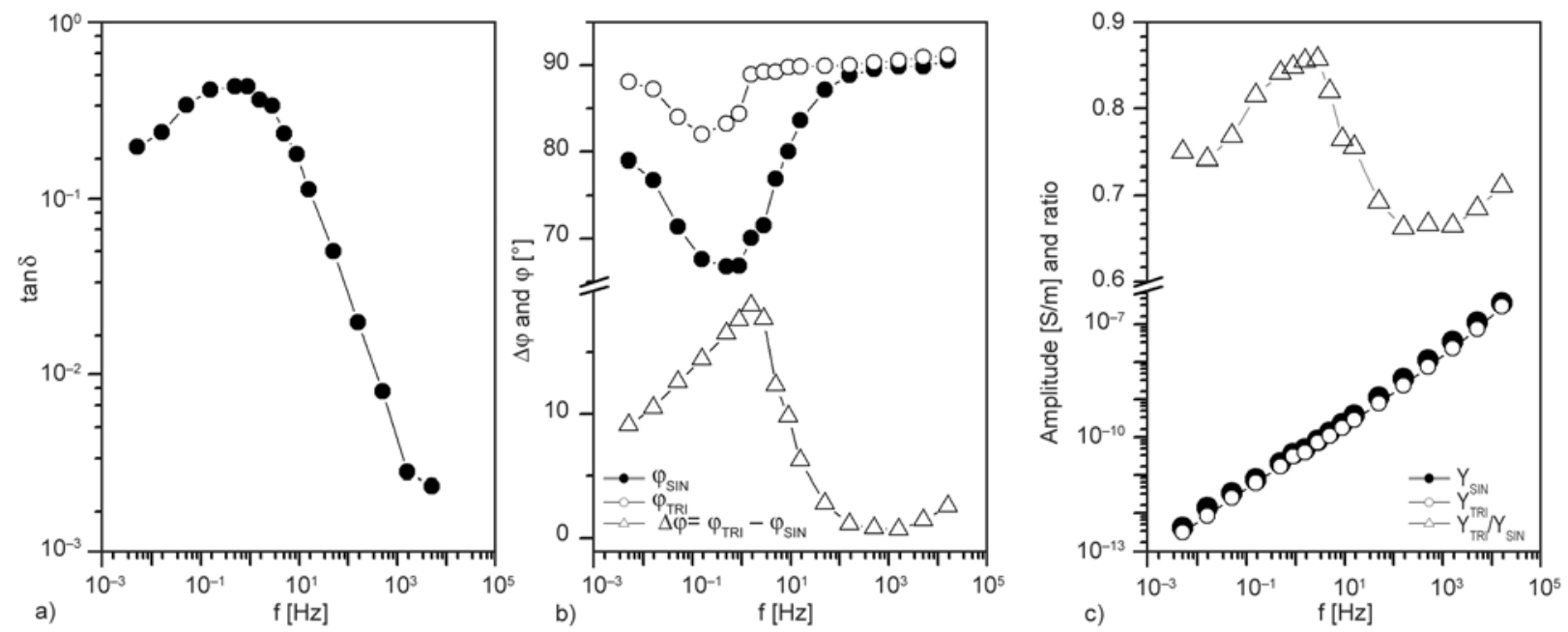

Figure 3. a) Loss tangent $\left(\tan \delta_{\mathrm{SIN}}\right)$, and the curves of b) differences in delay angles and c) amplitude ratios for polyvinyl chloride (PVC). The results were obtained experimentally by application of sinusoidal and triangular signals to the sample. 

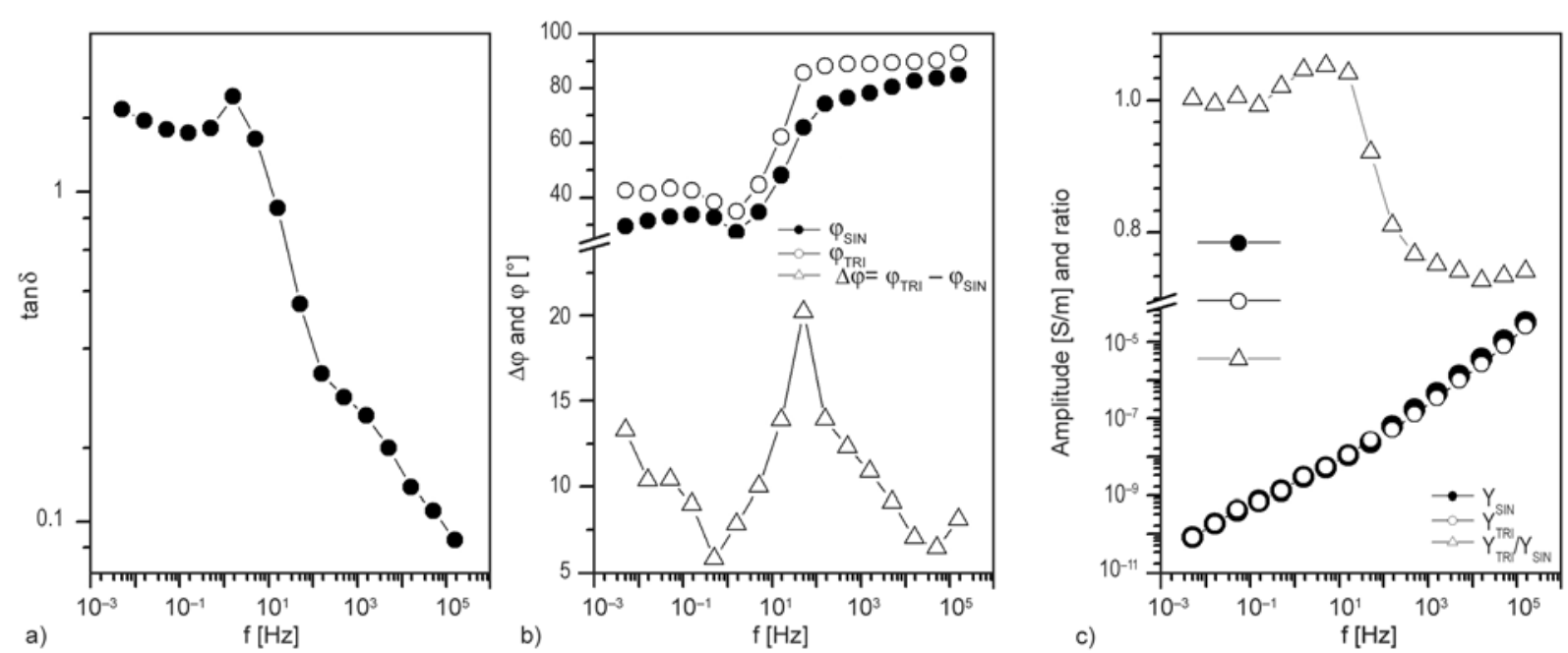

Figure 4. a) Loss tangent $\left(\tan \delta_{\mathrm{SIN}}\right)$, and the curves of b) differences in delay angles and c) amplitude ratios for polyvinyl alcohol (PVA). The results were obtained experimentally by application of sinusoidal and triangular signals to the sample.

shows a peak around $1 \mathrm{~Hz}$ attributable to the $\beta$ relaxation [20]. In addition to the $\beta$ relaxation, the curve of the differences in delay angles $\left(\Delta \varphi=\varphi_{\mathrm{TRI}}-\varphi_{\mathrm{SIN}}\right)$ also exhibits a peak at frequency $f_{0} \sim 1.5 \mathrm{~Hz}$ (Figure $3 b$ ) in the observed frequency range in PVC sample, as was suggested by the mathematical considerations on RC circuits (Figures 9a and 10a). The ratio between the amplitudes of conductivity at low frequencies is lower than $1\left(Y_{\mathrm{TRI}} / Y_{\mathrm{SIN}}<1\right)$ and has a peak at about $0.9 \mathrm{~Hz}$ (Figure 3c).

The loss tangent of PVA (Figure 4a) shows a small maximum at $1.5 \mathrm{~Hz}$. A peak is clearly observable in the curve of angle differences $\left(\Delta \varphi=\varphi_{\text {TRI }}-\varphi_{\text {SIN }}\right)$ with the maximum positioned at $f_{0} \sim 50 \mathrm{~Hz}$ (Figure $4 \mathrm{~b}$ ). The ratios of the amplitudes at low frequencies are close to 1 and the peak is at $\sim 5 \mathrm{~Hz}$ (Figure $4 \mathrm{c}$ ). The best agreement with the parallel RC circuit model was noticed in the composite of LDPE filled with $20 \%$ of carbon black. The loss tangent of the LDPE $+\mathrm{CB}$ composite shows a nearly linear dependence on frequency in the log-log scale (Figure 5a). The curve of the differences between the delay angles obtained by using sinusoidal and triangular excitation (Figure 5b) is similar to the results obtained mathematically on the parallel RC circuit (Figure $9 \mathrm{a}$ ). Figure $5 \mathrm{c}$ also shows that the ratio of the amplitudes of conductivity corresponds well with the results in Figure 9b. The characteristic frequencies obtained for the composite were $f_{0} \sim 5 \mathrm{kHz}$ and about $500 \mathrm{~Hz}$ for the maximum in amplitude ratio. The RC time constants of the samples estimated according to the model of a parallel $\mathrm{RC}$ circuit
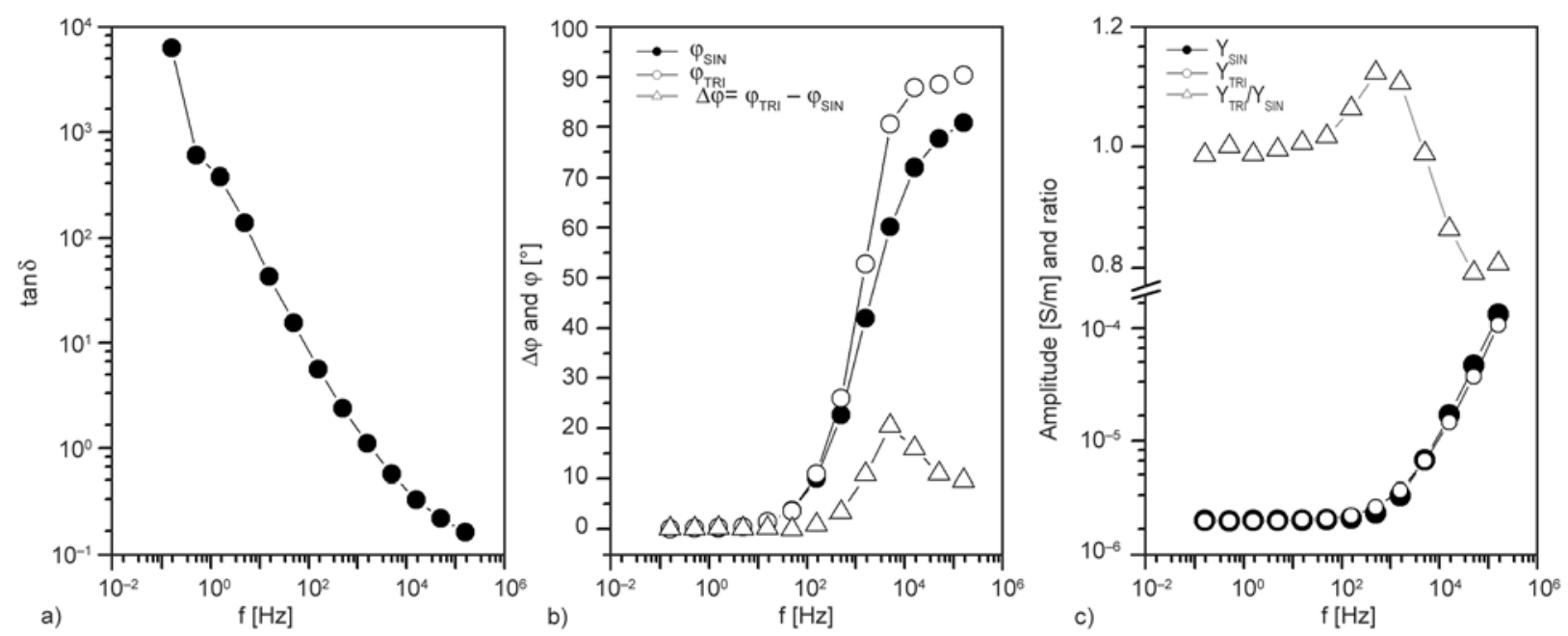

Figure 5. a) Loss tangent $\left(\tan \delta_{\text {SIN }}\right.$ ), and the curves of b) differences in delay angles and c) amplitude ratios for the composite LDPE filled with $20 \%$ of carbon black. The results were obtained experimentally by application of sinusoidal and triangular signals to the sample. 
(Table 2) and the corresponding DC conductivities are shown in Table 1. As can be seen, as the DC conductivity of the polymers or composites increases, the value $f_{0}$ also increases, which is in accordance with the established dependence of this quantity in terms of the idealized models of polymers according to the parallel and serial RC circuits $\left(f_{0} \sim 1 / R\right)$. The mentioned models also point to a more complex dependence, e.g. $f_{0} \sim 1 /(R C)$ (Table 2$)$. It is obvious that this parameter shows the biggest sensitivity to changes in polymer properties that are subject both to an increase in conductance (resistance decrease) and a decrease in capacity, or vice versa. A good example of this is the process of grouping the conductive particles in conductive paths in polymer composites, during which part of the capacitive contact switches to direct contact, giving rise to a decrease in capacity and an increase in conductance. Since the true $\varphi_{\operatorname{SIN}}=f(R C, \omega)$, the same is true for the sensitivity of the delay angle (or $\left.\tan \left(\pi / 2-\varphi_{\text {SIN }}\right)\right)$. However, $f_{0}$ is an integral parameter that does not depend on the frequency and describes the dielectric properties of the material in a wider range of frequencies. This is probably one of the reasons why it is more sensitive to changes in the dielectric properties of the material then the delay angle. It should be noted that for the detection of processes with opposite changes in resistance and capacitance in polymer materials $(R C \approx$ const), the admittance (or impedance) amplitude may be more suitable than the delay angle.

The results in Figures 3, 4 and 5 clearly show that application of sinusoidal and triangular signals enables one to draw additional information about the dielectric properties of real polymers samples. The curve of differences in delay angles $(\Delta \varphi=$ $\left.\varphi_{\text {TRI }}-\varphi_{\text {SIN }}\right)$ shows a well defined peak, even when the loss tangent implies more complex dielectric properties for a particular polymer. It should be noted that the methodology presented here may be used to also study other physical properties of poly-

Table 1. Experimentally determined $f_{0}$ and RC time constants calculated by the model of a parallel RC circuit (Table 3), and DC conductivities of the polymers and composite

\begin{tabular}{|l|c|c|c|}
\hline \multicolumn{1}{|c|}{ Sample } & $\begin{array}{c}\boldsymbol{f}_{\mathbf{0}} \\
{[\mathbf{H z}]}\end{array}$ & $\begin{array}{c}\boldsymbol{R} \boldsymbol{C}=\mathbf{1} / \mathbf{4} \boldsymbol{f}_{\mathbf{0}} \\
{[\mathbf{s}]}\end{array}$ & $\begin{array}{c}\boldsymbol{\sigma}_{\mathbf{D C}} \\
{[\mathbf{S} / \mathbf{m}]}\end{array}$ \\
\hline PVC & 1.58 & 0.156 & $1.2 \cdot 10^{-15}$ \\
\hline PVA & 50 & 0.005 & $3.3 \cdot 10^{-12}$ \\
\hline LDPE+CB & 5000 & 0.00005 & $2.1 \cdot 10^{-6}$ \\
\hline
\end{tabular}

mers. For example, it has been shown that electroluminescence of polymers induced by AC electrical signals exhibits a time shift, depending on the waveform signal applied [13].

\subsection{Changes in the dielectric properties of the LDPE + graphite composite induced by ageing}

As was noted previously, the frequency $\left(f_{0}\right)$ which corresponds to the maximum of the $\Delta \varphi(f)=$ $\varphi_{\text {TRI }}-\varphi_{\text {SIN }}$ curve could be a suitable parameter to describe the changes in the electrical properties of polymers. As an example of this, Figure 6 shows the effect of gamma irradiation on the dielectric properties of an LDPE + graphite composite. Radiationinduced processes of oxidation, networking and grafting in the composite increases its electrical conductivity [21], and these changes in the composite also affect the maximum of the $\Delta \varphi(f)$ (Figure 6a) and tangent loss (Figure 6b) curves. The dominant effect of a gamma radiation dose of about $100 \mathrm{kGy}$ is the networking of chains in the polyethylene matrix. The application of higher doses (200 kGy) leads to significant degradation of the crystal domains, in addition to networking, which is reflected by the electrical [21] and the mechanical properties [22]. The results shown in Figure 6 are in line with this influence of gamma radiation on the LDPE + carbon based composites. Unlike the composite samples exposed to gamma radiation in air, the physical and chemical changes in the composite samples that were aged at room temperature in high pressure oxygen for several days were significantly less.

The use of elevated temperature and oxygen pressure (thermo-oxidative ageing) is a frequently applied method of accelerated ageing of the polymeric materials to predict their lifetime. The degradation mechanism of thermo-oxidative ageing can be described through physical changes, densification and shrinkage, that are directly related to the thermo-mechanical history of the material, as well as surface chemical degradation with the formation of microcracks, and chemical changes in the sample [23]. Thermooxidative ageing usually causes degradation of mechanical properties but in some cases, such as reticulated resins, can lead to an improvement in these properties due to the subsequent process of networking [24]. The changes in electrical characteristics caused by these processes are very complex and diverse [6-9]. In the existing literature 

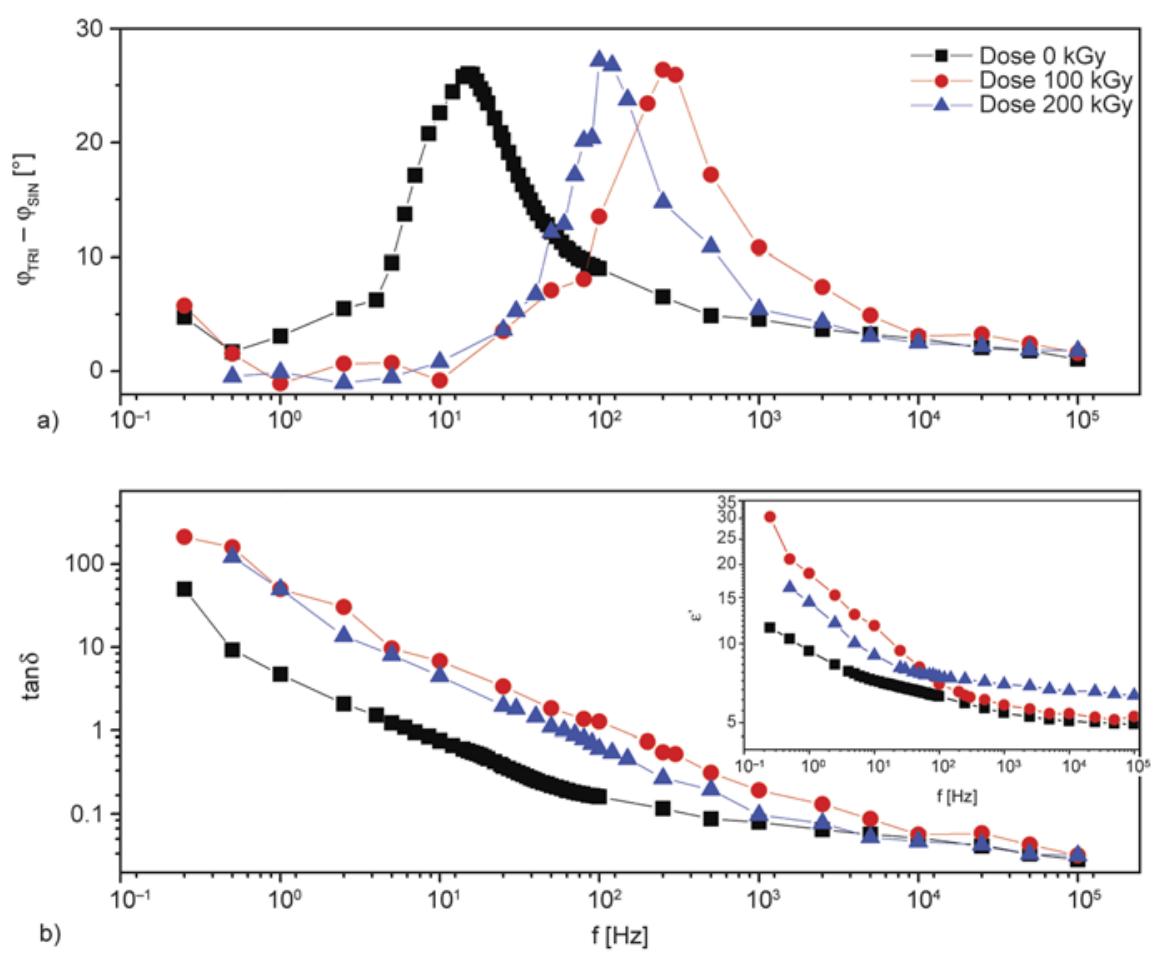

Figure 6. The effect of gamma irradiation on the dielectric properties of the LDPE+graphite composite: a) the curves of differences in delay angles and b) loss tangent $\left(\tan \delta_{\text {SIN }}\right)$ and permittivity (inset)

there is no evidence that the accelerated ageing that we applied to the LDPE + graphite composite (up to 4 days at room temperature ageing at high oxygen pressure) caused noticeable chemical or physical changes. We also could not detect such changes in the permittivity and loss tangent curves of these samples outside the experimental error $( \pm 1 \%)$. However, in the curves of the difference between the delay angles the initial ageing stage of the composites is clearly visible.

Figure 7a shows the frequency dependence of loss tangent and $\Delta \varphi$ of the LDPE + graphite composite which was subjected for different periods up to 4 days to oxygen at a high pressure at room temperature. While the loss tangent shows no significant differences due to the effect of ageing, there is a clear shift in the position of the maximum in the $\Delta \varphi(f)$ curves. The magnified part of Figure $7 \mathrm{a}$ is presented in Figure 7b. We emphasize that both the delay angles ( $\varphi_{\text {TRI }}$ and $\left.\varphi_{\text {SIN }}\right)$ were measured in the same experimental setup and with the same relative error of about $1 \%$. The results suggest that the position of the maximum in the $\Delta \varphi(f)=\varphi_{\text {TRI }}-\varphi_{\text {SIN }}$ curves ( $\varphi_{\text {TRI }}$ and $\varphi_{\text {SIN }}$ are measured by applying tri-
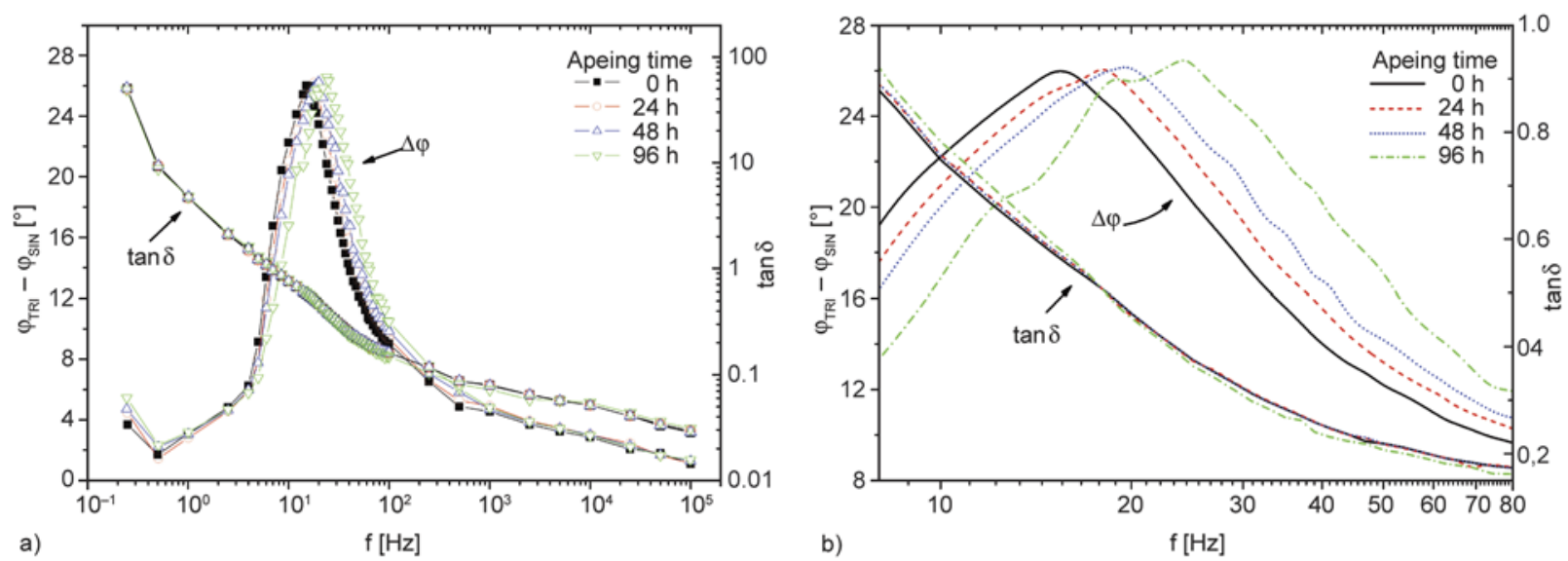

Figure 7. The effect of accelerated ageing on the dielectric properties of the LDPE+graphite composite: a) loss tangent $\left(\tan \delta_{\mathrm{SIN}}\right)$ and $\Delta \varphi ;$ b) the magnified part of the picture in a) 

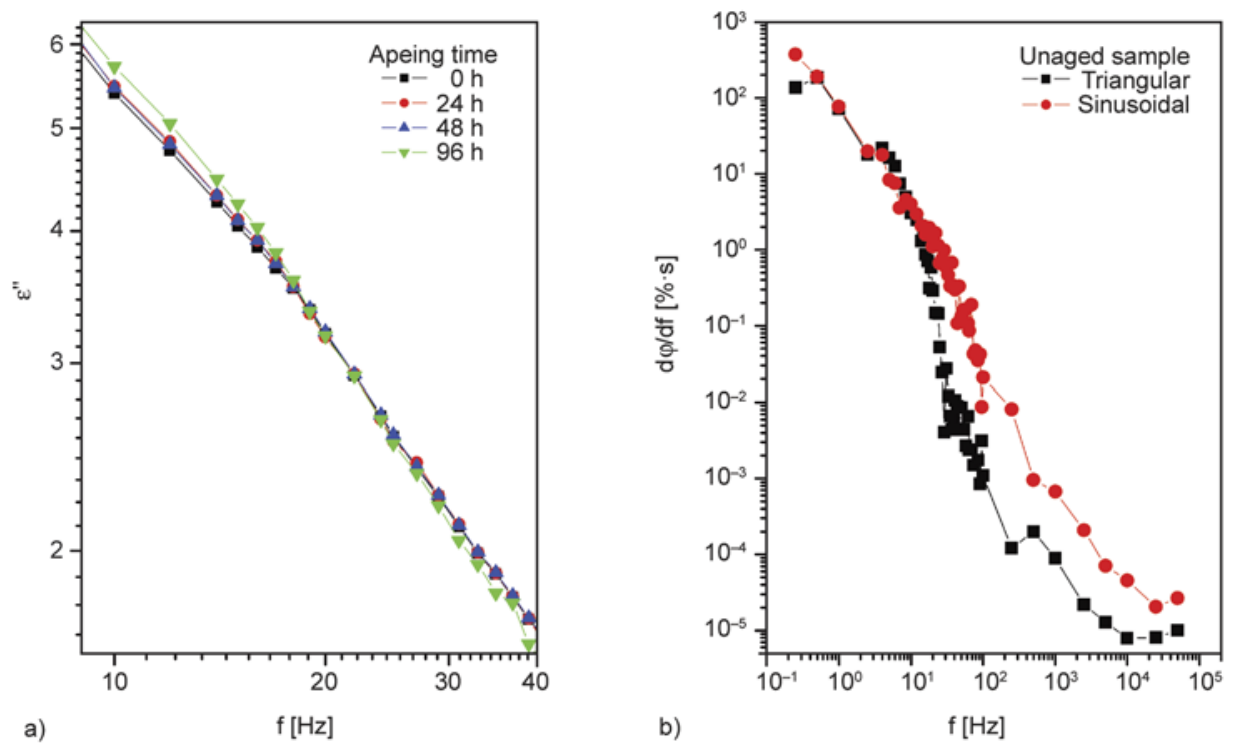

Figure 8. a) Imaginary part of permittivity of aged composite samples; b) normalised derivation of delay angles per frequency for unaged sample

angular and sinusoidal voltage respectively) shows more sensitivity to changes in the electrical properties of LDPE + graphite, due to ageing, than other dielectric parameters. These changes could also not be observed in the values of permeability (Figure $8 \mathrm{a}$ ), and there is also no evidence of these changes in the dependence of the derivation of the delay angles versus frequency (Figure $8 \mathrm{~b}$ ), because of big errors in these results.

As previously stated, there is no indication that the ageing treatment that we applied causes significant physical and chemical changes in the LDPE + graphite composite. In addition to the possible oxidation process, we believe that the observed shift in the value of $f_{0}$ is a consequence of compressive pressure and thereby induced structural relaxation in the samples during treatment rather than chemical changes. Bearing in mind the modeled dependence $f_{0} \sim 1 /(R C)$ (Table 2 ), the analysis of only this parameter cannot give conclusive evidence on whether the increase in $f_{0}$ with ageing time resulted from an increased number and/or quality of the direct contacts between the graphite particles in LDPE. However, based on existing experience such an increase is expected [8]. Since the change in amplitude of the conductivity caused by the oxygen ageing is also within experimental error, only one conclusion can be drawn, and that is that the observed increase in $f_{0}$ with increasing ageing time indicates a decrease in the delay angle $\varphi_{\text {SIN }}$ (parallel $\mathrm{RC}$ circuit), i.e. an increase in $\delta$ (and also $\tan \delta$ ) in the frequency spectrum. This can be concluded on the basis of the dependence $f_{0} \sim 1 /(R C)$ and the expression for $\varphi_{\text {SIN }}$ under the condition $\omega=$ const in the model of a parallel RC circuit (Table 2). The same result is obtained by considering $f_{0}$ and the delay angle in the model serial RC circuit.

\section{Conclusions}

We investigated the dielectric properties of different polymer systems with respect to the application of sinusoidal and triangular input signals. Our study also describes the possibility of dielectric characterization of the initial stages of ageing in a low-density polyethylene + graphite composite using comparison of the delay angles obtained by triangular and sinusoidal voltage excitations. The differences in the results obtained with these two signal excitations were first studied by simulation on parallel and serial RC circuits (see Appendix). The curve of the differences in delay angles $\left(\Delta \varphi=\varphi_{\text {TRI }}-\varphi_{\text {SIN }}\right)$ obtained by the differently shaped input signals shows a peak with a well defined position of the maximum. The frequency that corresponds to this maximum can be related to the RC time constant $(R C)$ of the circuits by the simple formulas $f \approx$ $1 /(4 R C)$ for a parallel and $f \approx 1 /(7.2 R C)$ for a serial $\mathrm{RC}$ circuit. All the tested polymers (PVC and PVA) and polymer composites (LDPE + CB and LDPE + graphite) show a well-defined peak in the $\Delta \varphi(f)$ spectra. Through the use of this method we discovered early ageing processes in an LDPE + graphite composite that could not be detected by conventional dielectric spectroscopy methods. Our results 
suggest that the position of the maximum in the $\Delta \varphi(f)=\varphi_{\mathrm{TRI}}-\varphi_{\mathrm{SIN}}$ curves $\left(\varphi_{\mathrm{TRI}}\right.$ and $\varphi_{\mathrm{SIN}}$ are measured by applying triangular and sinusoidal voltage respectively) is more sensitive to changes in the electrical properties of polymer materials then angle $\delta$ (or $\tan \delta$ ). We think that the proposed methodology could find industrial application, for example, in early stage detections of ageing in electrical insulation and transformer oils or for the purpose of monitoring other physical or chemical changes in materials.

\section{Appendix}

The basic equations that characterise the parallel and serial RC circuit in terms of the application of the sinusoidal and triangular signals are given in Table 2. AC currents $i(t)$, that are shown in Table 2, are obtained by applying the differential equations for the RC circuits on the expression for the triangular wave voltage which are also presented in the same table.

The analysis of the responses of serial and parallel $\mathrm{RC}$ circuits was done by using software that was specifically designed for this purpose, and some of these results were checked by measurements on different RC circuits, which confirms the validity of the applied equations and software operation.

Two basic parameters were studied: the amplitude of AC conductivity $(Y)$ or AC resistivity $(Z)$ and delay angle $(\varphi)$ (see Figure 2). The amplitudes $(Y$ and $Z$ ) and delay angles (for both applied waveforms) were presented for four different values of $R$ and $C$, but keeping their product constant in Figure 9 for parallel and in Figure 10 for serial RC circuits $(R C(1), R C(2), R C(3)$ and $R C(4)$ are presented in Table 3). As for a sinusoidal signal (Table 2), the delayed angle upon triangular excitation on serial and parallel RC circuits depended on the time constant but not on the individual values of $R$ and $C$. For this reason we presented only the results for $R C(1)$ in Figures9a and 10a. It is clear that the delayed angles for these two signals differ in the narrow range of intermediate frequencies (around $1 \mathrm{kHz}$ ), while their difference, $\Delta \varphi=\varphi_{\mathrm{TRI}}-\varphi_{\text {SIN }}$, exhibits a peak of about $33^{\circ}$ for parallel and $9^{\circ}$ for serial circuits at the corresponding frequencies $f_{0}$. It was found that this frequency can be related to the circuit time constant $(R C)$ by the empirical formulas shown in Table 2. The results shown in Figures 9, 10 and 11 were also verified by experimental measurements.

The amplitudes of admittance (AC conductivity) of both signals for the parallel RC circuit (Figure 9b) are almost inseparable at low frequencies $(f<<$

Table 3. RC circuits and corresponding parameters for dielectric analyses presented in Figures 9, 10 and 11

\begin{tabular}{|c|c|c|c|}
\hline $\mathbf{N}^{\circ}$ & $\begin{array}{c}\boldsymbol{R} \\
{[\boldsymbol{\Omega}]}\end{array}$ & $\begin{array}{c}\boldsymbol{C} \\
{[\boldsymbol{\mu} \mathbf{F}]}\end{array}$ & $\begin{array}{c}\boldsymbol{R C} \\
{\left[\mathbf{1 0}^{-4} \mathbf{s}\right]}\end{array}$ \\
\hline 1 & 1737.5 & 0.1036 & 1.80005 \\
\hline 2 & 1491.5 & 0.1207 & 1.80069 \\
\hline 3 & 1191.5 & 0.1509 & 1.79881 \\
\hline 4 & 978.5 & 0.1839 & 1.79946 \\
\hline 5 & 2990.1 & 0.0386 & 1.15414 \\
\hline 6 & 703.5 & 0.3271 & 2.30044 \\
\hline
\end{tabular}

Table 2. The corresponding equations for parallel and serial $R C$ circuit

\begin{tabular}{|c|c|c|}
\hline & Parallel RC circuit & Serial RC circuit \\
\hline Differential equations & $i(t)=\frac{u(t)}{R}+C \frac{\mathrm{d} u(t)}{\mathrm{d} t}$ & $i(t)=C \frac{\mathrm{d} u_{\mathrm{C}}(t)}{\mathrm{d} t}=\frac{u_{\mathrm{R}}(t)}{R}$ \\
\hline $\begin{array}{l}\text { Delay angle upon sinusoidal } \\
\text { coercion }\end{array}$ & $\varphi_{\mathrm{SIN}}=\frac{\pi}{2}-\arctan \frac{1}{R C \omega}$ & $\varphi_{\mathrm{SIN}}=\frac{\pi}{2}-\arctan R C \omega$ \\
\hline $\begin{array}{l}\text { Amplitude of admittance or } \\
\text { impedance }\end{array}$ & $Y_{\mathrm{SIN}}=\sqrt{\frac{1}{R^{2}}+(C \omega)^{2}}$ & $Z_{\mathrm{SIN}}=\sqrt{R^{2}+\left(\frac{1}{C \omega}\right)^{2}}$ \\
\hline Triangular signal & $u(t)=\frac{8 u_{0}}{\pi^{2}} \sum_{\mathrm{k}=0}^{\infty}$ & $\frac{\mathrm{os}[(2 k+1) \omega t]}{(2 k+1)^{2}}$ \\
\hline \multirow{2}{*}{$\begin{array}{l}i(t)-\text { Current in circuit upon } \\
\text { triangular coercion }\end{array}$} & $8 u_{0} \sum^{\infty} \cos [(2 k+1) \omega t]-R C \omega(2 k+1) \sin [(2 k+1) \omega t]$ & $\frac{8 u_{0}}{2} \sum^{\infty} \cos [(2 k+1) \omega t]-\frac{1}{R C \omega(2 k+1)} \sin [(2 k+1) \omega t]$ \\
\hline & $\overline{R \pi^{2}} \sum_{\mathrm{k}=0} \overline{(2 k+1)^{2}}$ & $\overline{R \pi^{2}} \sum_{\mathrm{k}=0} \overline{(2 k+1)^{2}+\left(\frac{1}{R C \omega}\right)^{2}}$ \\
\hline $\begin{array}{l}f_{0}-\text { Frequency that corre- } \\
\text { sponds to a maximum differ- } \\
\text { ence between delay angles, } \\
\varphi_{\text {TRI }}-\varphi_{\text {SIN }} \text { in range } \\
f_{0} \in(0.01 \mathrm{~Hz}, 100 \mathrm{kHz})\end{array}$ & $\frac{1}{f_{0} R C}=4.06 \pm 0.03 \Rightarrow f_{0} \approx \frac{1}{4 R C}$ & $\frac{1}{f_{0} R C}=7.2 \pm 0.08 \Rightarrow f_{0} \approx \frac{1}{7.2 R C}$ \\
\hline
\end{tabular}



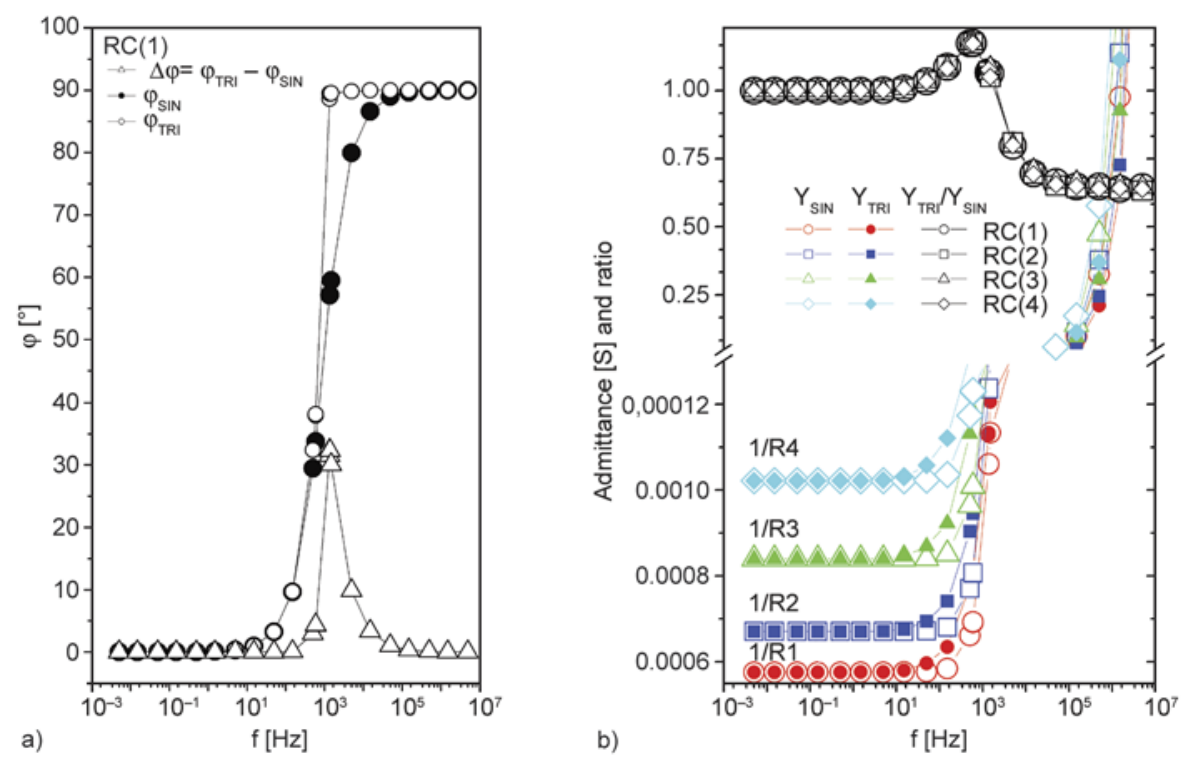

Figure 9. a) Delay angles and b) the amplitudes of admittance obtained by software simulation on parallel RC circuits with the four equal time constants $R C$ ( $R$ and $C$ values were altered and stated in Table 3 ). In the case of delay angles, the results for $R C(1)$ were presented, since the calculations with other time constants gave identical values.
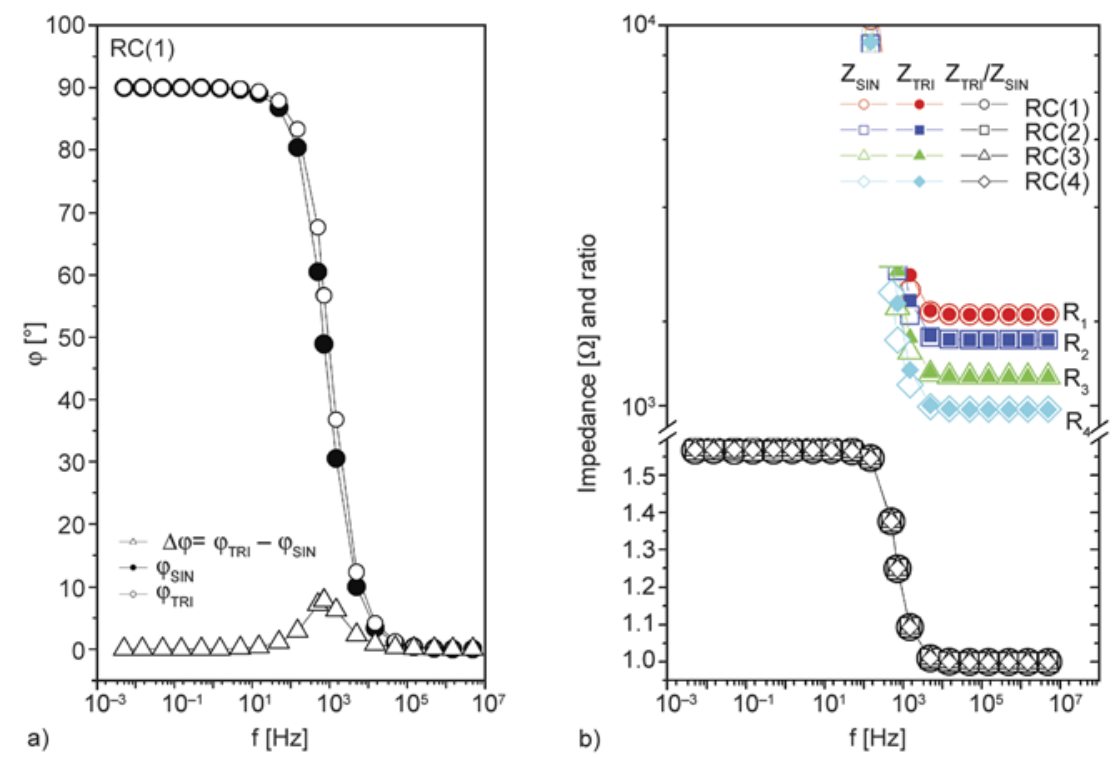

Figure 10. a) Delay angles and b) the amplitudes of impedance obtained by software simulation on serial RC circuits with the four equal time constants $R C$ ( $R$ and $C$ values were altered and stated in Table 1$)$. In the case of delay angles, the results for $R C(1)$ were presented, since the calculations with other time constants gave identical values.

$1 / R C)$ and $Y_{\mathrm{TRI}}=Y_{\mathrm{SIN}}=1 / R$ for each particular resistivity. However, they do differ at higher frequencies $(f>>1 /(R C))$, with the $Y_{\mathrm{TRI}} / Y_{\mathrm{SIN}}$ ratio approaching $\sim 0.64$. In the case of the impedance amplitude in serial RC circuits the relation $Z_{\mathrm{TRI}}=Z_{\mathrm{SIN}}=R$ at $f>>$ $1 /(R C)$ is also true (Figure $10 \mathrm{~b}$ ).

Figure 11 shows the values of the differences in the delay angles and amplitude ratios for both the input signals calculated (for the parallel RC model) by using the different time constants $R C(3), R C(5)$ and $R C(6)$ from Table 3, as well as the loss tangent for the sinusoidal signal calculated for the same con- stants. Figure 11a shows that the dependence of tan $\delta\left(\delta=\pi / 2-\varphi_{\mathrm{SIN}}\right)$ on frequency is a straight line in the $\log -\log$ scale with a slope equal to -1 . At the same time, the curves of $Y_{\mathrm{TRI}} / Y_{\mathrm{SIN}}$ and $\Delta \varphi$ exhibit maxima that shift towards lower frequencies with increasing time constant. Similar results were obtained for the case of differences in delay angles for a serial RC circuit.

\section{Acknowledgements}

This work was supported by the Ministry of Education and Science, Republic of Serbia (Project No. 171029). 

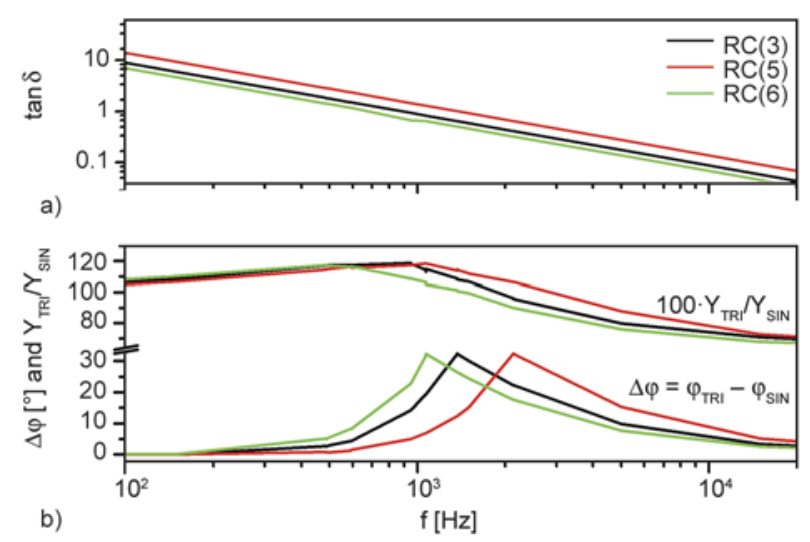

Figure 11. The results obtained by using different time constants: $R C(4), R C(5)$ and $R C(6)$ in Table 3 on parallel RC circuit. a) Loss tangent $(\tan \delta)$ and b) the curves of differences in delay angles and amplitude ratios (as indicated in figure).

\section{References}

[1] Struik L. C. E.: The mechanical and physical ageing of semicrystalline polymers: 1 . Polymer, 28, 1521-1533 (1987).

DOI: 10.1016/0032-3861(87)90353-3

[2] Wiles M. D., Scott G.: Polyolefins with controlled environmental degradability. Polymer Degradation and Stability, 91, 1581-1592 (2006).

DOI: $10.1016 /$ j.polymdegradstab.2005.09.010

[3] Dudić D., Djoković V., Kostoski D.: The high temperature secondary crystallisation of aged isotactic polypropylene. Polymer Testing, 23, 621-627 (2004). DOI: $10.1016 /$ j.polymertesting.2004.01.015

[4] Wu Q., Qu B., Xu Y., Wu Q.: Surface photo-oxidation and photostabilization of photocross-linked polyethylene. Polymer Degradation and Stability, 68, 97-102 (2000).

DOI: 10.1016/S0141-3910(99)00171-8

[5] Djoković V., Kostoski D., Dramićanin M., Dudić D.: Effects of gamma irradiation on the stress relaxation of drawn ultrahigh molecular weight polyethylene. Radiation Physics and Chemistry, 55, 605-607 (1999).

DOI: 10.1016/S0969-806X(99)00255-8

[6] Sakamoto W. K., Perez D. J., Das-Gupta D. K.: On the characterisation of electrically stressed polyethylene before and after chemical treatment. Journal of Materials Science, 37, 1295-1300 (2002).

DOI: 10.1023/A:1014547907534

[7] Simonović K., Petronijević I., Kostoski D., Dojčilović J., Luyt S. A., Dudić D.: Effects of acid treatment at different temperatures on the surface dielectric properties of low-density polyethylene. Polymer International, in press (2014).

DOI: $10.1002 /$ pi.4731
[8] Vu Q. D., Gigliotti M., Lafarie-Frenot C. M.: Experimental characterization of thermo-oxidation-induced shrinkage and damage in polymer-matrix composites. Composites Part A: Applied Science and Manufacturing, 43, 577-586 (2012).

DOI: 10.1016/j.compositesa.2011.12.018

[9] Sun Y., Luo S., Watkins K., Wong P. C.: Electrical approach to monitor the thermal oxidation aging of carbon black filled ethylene propylene rubber. Polymer Degradation and Stability, 86, 209-215 (2004). DOI: $10.1016 /$ j.polymdegradstab.2004.04.013

[10] Hedvig P.: Dielectric spectroscopy of polymers. Akadémiai Kiadó, Budapest (1977).

[11] Asami K.: Characterization of heterogeneous systems by dielectric spectroscopy. Progress in Polymer Science, 27, 1617-1659 (2002). DOI: $10.1016 / \mathrm{S} 0079-6700(02) 00015-1$

[12] Boyd H. R.: Relaxation processes in crystalline polymers: Experimental behaviour - A review. Polymer, 26, 323-347 (1985).

DOI: 10.1016/0032-3861(85)90192-2

[13] Baudoin F., Mills D. H., Lewin P. L., Le Roy S., Teyssedre G., Laurent C.: Modeling electroluminescence in insulating polymers under AC stress: Effect of excitation waveform. Journal of Physics D: Applied Physics, 44, 165402/1-165402/11 (2011). DOI: $10.1088 / 0022-3727 / 44 / 16 / 165402$

[14] Suwarno: Role of applied voltage waveforms on partial discharge patterns of electrical treeing in low density polyethylene. International Journal of Electrical and Power Engineering, 3, 184-190 (2009).

[15] Janssen R. P. M., Govaert L. E., Meijer H. E. H.: An analytical method to predict fatigue life of thermoplastics in uniaxial loading: Sensitivity to wave type, frequency, and stress amplitude. Macromolecules, 41, 2531-2540 (2008).

DOI: $10.1021 / \mathrm{ma} 071274 \mathrm{a}$

[16] Gasulla-Forner M., Jordana-Barnils J., Pallas-Areny R., Torrents J. M.: Subsurface resistivity measurements using square waveforms. IEEE Transactions on Instrumentation and Measurement, 47, 74-77 (1998). DOI: $10.1109 / 19.728793$

[17] Bailey J. J.: The triangular wave test for electrocardiographic devices: A historical perspective. Journal of Electrocardiology, 37, 71-73 (2004).

[18] Creason S. C., Hayes J. W., Smith D. E.: Fourier transform faradaic admittance measurements III. Comparison of measurement efficiency for various test signal waveforms. Journal of Electroanalytical Chemistry and Interfacial Electrochemistry, 47, 9-46 (1973). DOI: $10.1016 / \mathrm{S} 0022-0728(73) 80343-2$

[19] Dudić D., Škipina B., Dojčilović J., Novaković L., Kostoski D.: Effects of charge trapping on the electrical conductivity of low-density polyethylene-carbon black composites. Journal of Applied Polymer Science, 121, 138-143 (2011). DOI: $10.1002 /$ app. 33421 
[20] Elícegui A., Del Val J. J., Millán L. J., Mijangos C.: $\alpha$ and $\beta$ relaxation processes in internally plasticized poly (vinylchloride). Journal of Non-Crystalline Solids, 235-237, 623-627 (1998).

DOI: $10.1016 / \mathrm{S} 0022-3093(98) 00664-4$

[21] Balabanov S., Krezhov K.: Electrical conductivity and electrostatic properties of radiationally modified polymer composites with carbon black. Journal of Physics D: Applied Physics, 32, 2573-2577 (1999).

DOI: $10.1088 / 0022-3727 / 32 / 19 / 315$

[22] Suarez J. C. M., Monteiro E. E. C., Mano E. B.: Study of the effect of gamma irradiation on polyolefins Low-density polyethylene. Polymer Degradation and Stability, 75, 143-151 (2002).

DOI: $10.1016 / \mathrm{S} 0141-3910(01) 00213-0$
[23] Tsotsis K. T., Keller S., Bardis J., Bish J.: Preliminary evaluation of the use of elevated pressure to accelerate thermo-oxidative aging in composites. Polymer Degradation and Stability, 64, 207-212 (1999). DOI: 10.1016/S0141-3910(98)00190-6

[24] Middleton J., Burks B., Wells T., Setters M. A., Jasiuk I., Kumosa M.: The effect of ozone and high temperature on polymer degradation in polymer core composite conductors. Polymer Degradation and Stability, 98, 2282-2290 (2013).

DOI: $10.1016 /$ j.polymdegradstab.2013.08.013 